\title{
Prescription refill, patient self-report and physician report in assessing adherence to oral endocrine therapy in early breast cancer patients: a retrospective cohort study in Catalonia, Spain
}

\section{R Font ${ }^{1,2}$, JA Espinas', M Gil-Gil ${ }^{3}$, A Barnadas ${ }^{4}$, B Ojeda ${ }^{4}$, I Tusquets $^{5}$, MA Segui ${ }^{6}$, M Margeli ${ }^{7}$, A Arcusa ${ }^{8}$, A Prat', M Garcia ${ }^{10}$ and JM Borras ${ }^{*, 1,2}$}

'Catalonian Cancer Strategy Unit, Department of Health, Catalonian Regional Authority, Gran Via de l'Hospitalet 199-203, L'Hospitalet de Llobregat, Barcelona 08908, Spain; ${ }^{2}$ Department of Clinical Sciences, Bellvitge Biomedical Research Institute, University of Barcelona. Feixa Llarga S/N, L'Hospitalet de Llobregat, Barcelona 08907, Spain; ${ }^{3}$ Department of Medical Oncology, Bellvitge Biomedical Research Institute (Institut d'Investigació Biomèdica de Bellvitge - IDIBELL), Catalonian Institute of Oncology (Institut Català d'Oncologia - ICO), Gran Via de l'Hospitalet 199-203, L'Hospitalet de Llobregat, Barcelona 08908, Spain; 'Department of Medical Oncology, Santa Creu i Sant Pau Hospital, Sant Antoni Maria Claret 167, Barcelona 08025, Spain; ${ }^{5}$ Department of Medical Oncology, Del Mar Hospital, Passeig Maríim 25-29, Barcelona 08003, Spain; ${ }^{6}$ Department of Medical Oncology, Parc Taulí Health Corporation (Corporació Sanitària Parc Tauli), Carretera de Prats de Lluçanés 166, Sabadell 08208, Spain; ' Department of Medical Oncology, Germans Trias i Pujol Hospital, ICO-Badalona, Carretera de Canyet s/n, Badalona 08916, Spain; ${ }^{8}$ Department of Medical Oncology, Terrassa Health Consortium (Consorci Sanitari de Terrassa), Torrebonica s/n, Terrassa 08227, Spain; ${ }^{9}$ Pharmaceutical Care and Complementary Benefits, Catalonia Health Service, Department of Health, Catalonian Regional Authority. Travessera de les Corts 131-159, Barcelona 08028, Spain; ${ }^{10}$ Cancer Prevention and Control Group, Bellvitge Biomedical Research Institute, IDIBELL, Catalonian Institute of Oncology. Gran Via de l'Hospitalet 199-203, L'Hospitalet de Llobregat, Barcelona 08908, Spain

AIMS: To compare different methods in order to assess adherence and persistence with oral endocrine therapy in women diagnosed with breast cancer (BC) in Catalonia.

MATERIALS AND METHODS: This study covered all women newly diagnosed with stage I, II or IIla BC and positive hormone receptors at six hospitals in Catalonia (Spain) in 2004. Adherence was assessed on the basis of physician report and patient self-report using a telephone questionnaire. Persistence was measured by refill prescriptions. We used the Kappa index to compare adherence measures and logistic regression to evaluate adherence-related risk factors.

RESULTS: The study covered a total of 692 women. Adherence ranged from 92\% (self-report) to 94.7\% (physician report), depending on the measure used; persistence was $74.7 \%$ at 5 years of follow-up. Low concordance between measures was observed (Kappa range: $0.018-0.267)$. Patients aged $50-74$ years showed higher adherence than those aged $<50$ years. Adherence was also associated with: adjuvant chemotherapy and sequential hormonal therapy.

CONCLUSIONS: Concordance between the different measures was remarkably low, indicating the need for further research. Adherence is an issue in the management of BC patients taking oral drugs, and should be assessed in clinical practice.

British Journal of Cancer (2012) 107, 1249-1256. doi:I0.1038/bjc.2012.389 www.bjcancer.com

Published online 6 September 2012

(C) 2012 Cancer Research UK

Keywords: early breast cancer; adherence; persistence; endocrine therapy

Breast cancer $(\mathrm{BC})$ is the most frequent cancer among women, accounting for $28.1 \%$ of all cancer diagnosed in Catalonia in the period 1998-2002 (Borras et al, 2009). Early detection, screening and improvements in therapy have increased the life expectancy and quality of life these patients (Berry et al, 2005). One such improvement is adjuvant treatment of BC patients, which, depending on the disease stage and other prognostic factors, could take the form of one or more of the following procedures: radiotherapy; chemotherapy; target therapy; and oral endocrine treatment. Clinical guidelines recommend that hormone receptorpositive $\mathrm{BC}$ patients receive at least 5 years of oral endocrine therapy (Manchon et al, 2010). Tamoxifen reduces risk of

*Correspondence: Dr JM Borras; E-mail: jmborras@iconcologia.net Received I 8 June 2012; revised 6 August 2012; accepted 7 August 2012; published online 6 September 2012 progression and death (Early Breast Cancer Trialist' Collaborative Group (EBCTCG), 2005; Lash et al, 2006). Aromatase inhibitors have demonstrated superiority to tamoxifen, whether alone or in sequence with tamoxifen in postmenopausal women with BC (Mouridsen et al, 2009).

One of the most relevant issues linked to chronic diseases is lack of adherence to therapy. Low adherence to treatment may influence its efficacy and increase health service use and associated costs (Haynes et al, 1977; Darkow et al, 2007). DiMatteo (2004) conducted a review of the studies published in the last 50 years and observed that average adherence for all types of disease and treatment was $76.2 \%$ overall. Although there are a number of different methods for assessing adherence to treatment, there is no consensus as to which could be considered a gold standard (Rudd, 1979). Direct methods are all objective but are costly and difficult to apply in clinical practice; and, while 
indirect methods, such as patient interviews, are less expensive and easy to implement, they can nonetheless be biased, usually towards overestimating adherence (Wang et al, 2004). Recently, the monitoring of prescribed, refilled and reimbursed medical treatment from community or hospital pharmacies has been used (Andersson et al, 2005), a methodology that has been applied in studies targeting BC patients (Nilsson et al, 2006). Behaviour related to drug taking has been divided into two distinct although closely related - concepts, namely, adherence and persistence. Whereas adherence refers to the frequency of drug use during therapy (how well a patient is taking the prescribed drug), persistence refers to the overall duration of therapy (how long a patient has been taking the drug) (Cramer et al, 2008).

Until recently, the problem of non-adherence was not a subject of major interest in cancer care, due mainly to the fact that almost all therapies were parentally administered in a hospital setting (though it should perhaps be mentioned here that non-adherence is also a problem in parenteral cancer drugs) (Borras et al, 2001). However, the recent development of oral therapies in oncology could mark a turning point in the perceived need for assessing adherence among cancer patients. Moreover, patients tend to prefer oral therapies to parenteral drugs (Liu et al, 1997; Fallowfield et al, 2006).

There were no data for southern Europe that would have enabled the extent of the problem posed by non-adherence to be assessed, by using a range of measures to analyse concordance among them. We carried out a 5-year follow-up study of patients first diagnosed with early BC in Catalonia in 2004. The main aim of this study was to assess the degree of adherence to endocrine adjuvant therapy, and the consistency between different measures of adherence, which were deemed feasible in daily clinical practice.

\section{MATERIALS AND METHODS}

\section{Study population}

A retrospective study was designed, including all new patients diagnosed with BC during 2004 at six hospitals in the city of Barcelona and the Greater Barcelona area (Catalonian Institute of Oncology-Duran i Reynals Hospital, Catalonian Institute of Oncology-Gemans Trias i Pujol, Santa Creu i Sant Pau Hospital, del Mar Hospital, Terrassa Health Consortium, Parc Taulí Health Corporation). Breast cancer patients were identified by means of hospital registries and the minimum basic data set for all patient discharges, which is mandatory in the Catalonian Health Service. All patients diagnosed at the participating health centres in 2004 were reviewed $(N=1133)$ and found that $61 \%$ of patients met the inclusion criteria, that is, case of histologically confirmed, incident, invasive BC in stages I, II or IIIa, with analysis of hormonal-positive receptors and at least one prescription for adjuvant endocrine treatment (Manchon et al, 2010). Information was collected between November 2009 and April 2010. The study protocol was reviewed and approved by the ethics committees of all the participating hospitals. Informed consent was obtained from all patients.

\section{Data sources and measures used}

Clinical records were reviewed to collect data on tumour characteristics, stage and hormone receptors (oestrogens and progesterone), along with any indication of adjuvant endocrine treatment, adherence as recorded in the clinical record, side effects recorded in patients' clinical records and patients' vital status. A telephone interview was conducted by trained personnel, using a standard structured questionnaire to gather information on adjuvant oral endocrine therapy, adherence and problems experienced in taking the medication as reported by the patient. Finally, data on the use of endocrine therapy for BC treatment over the study period (2004-2009) was drawn from the community pharmacy database of refill prescriptions, which is mandatory for reimbursement of the therapies in Spain.

For study purposes, treatment persistence and adherence were deemed to be outcomes. As proposed by Cramer et al (2008), adherence was defined as 'the extend to which a patient acts in accordance with the prescribed interval and dose of a dosing regimen' and persistence as 'the duration of time from initiation to discontinuation of therapy'. Discontinuation of therapy was considered when prescription was not refilled for more than 2 months. A change of drug was considered as a continuation of treatment. The study follow-up time was 5 years from the date of the first prescription refill. Where recurrence or death occurred, this was taken as the last date for persistence analysis.

Three different data sources were used, namely:

Physician report Adherence was measured as reported by patients' physicians in their clinical records.

Telephone questionnaire To measure adherence, all patients were telephonically interviewed using the following question, 'How often do you have difficulties in taking your medicine or do you ever forget to take it?', to which possible answers were, 'never', 'sometimes', 'quite a lot', 'all the time' and 'I do not remember'. One variable was defined to assess level high of adherence with women who answered 'never' or 'sometimes'.

Administrative drug-reimbursement database This is a mandatory register of all refilled prescriptions at pharmacies requiring reimbursement by the Catalonian Health Service. All patients included had received treatment at a public hospital, and as a result the database furnished full coverage on such cases. Treatment persistence was estimated as the proportion of days covered during the prescribed treatment period. Data were computed up to a maximum of 5 years from the date of the first prescription refill, thus it could be considered a cumulative 5 years measure of adherence. Both persistence and adherence was deemed satisfactory if coverage of the prescribed medication was 80-110\% (Hershman et al, 2010b).

\section{Statistical analysis}

The outcome obtained from the three different data sources were assessed individually. Persistence was transformed in categorical variable in accordance to the $80-110 \%$ coverage of the prescribed medication to be comparable with the other outcomes. Adherence is used the outcome variable with the three different sources of information collected in this study univariate comparison between categorical and dependent variables was performed using a $\chi^{2}$-test. Unconditional logistic regression was applied to estimate the odds ratios (OR) and 95 per cent confidence intervals (CI, 95\% CI) of non-adherence to oral hormone therapy vis-à-vis different factors, with adherent or persistent patients taken as the reference group. Models were adjusted for age $(<50,50-74$ and $>74$ years old were the categories applied), hospital and those variables associated in the univariate analysis $(P<0.1)$ for each dependent measure of adherence. Concordance among the three methods used to assess adherence was estimated by means of the Kappa index (Landis and Koch, 1977). The level of significance was set at $5 \%$ and all tests were two-tailed. Adverse effects were analysed as a dichotomous variable reporting the presence or absence of them for individual patients. All analyses were performed using the SPSS (version 13.0, September 2004) software programme.

\section{RESULTS}

A total of 692 women fulfilled the inclusion criteria (Figure 1). Descriptive demographic and clinical data on all patients in the 


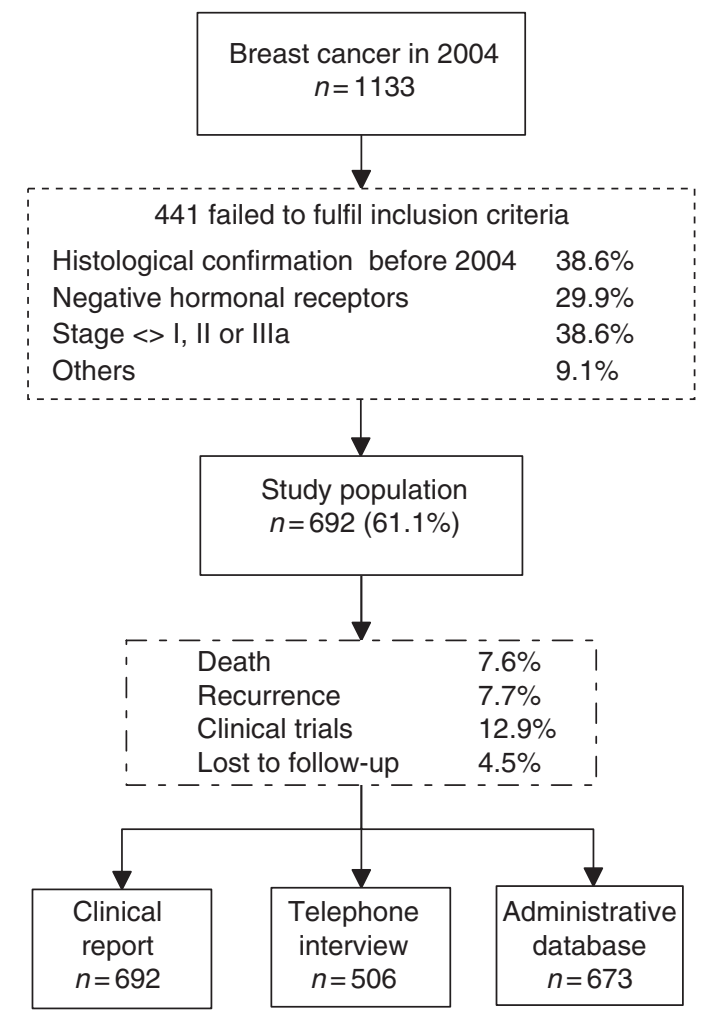

Figure I Flow chart of participants.

study sample are shown in Table 1 . The main surgical treatment was conservative breast surgery (79\%). In all, $89 \%$ thus received chemotherapy and/or radiotherapy. The most frequent endocrine therapies were tamoxifen and anastrozole as one-drug treatment, and tamoxifen followed by exemestane as sequential therapy. Disease progression was observed in $8 \%$ of cases.

Although the lowest adherence was observed for the Administrative drug-reimbursement database $(74.7 \%$ adjusted for age, centre and stage), the highest adherence was observed for physician report (94.7\%) (Table 2). Concordance among the three methods was very low (range: -0.018 to 0.267 ), showing an almost non existent consistency in the reports of adherence by the three measures used.

Table 3 showed the multivariate analysis of the different measures of treatment adherence and their association with the potential explanatory variables. Insofar as physician-assessed adherence was concerned, age was associated with adherence, with women aged 50-74 years and over being more likely to be adherents $(\mathrm{OR}=1.71,95 \% \mathrm{CI}: 0.72-4.06$ and $\mathrm{OR}=2.56,95 \% \mathrm{CI}$ : $0.68-9.63$, respectively) than those aged under 50 years. Furthermore, adjuvant chemotherapy $(\mathrm{OR}=2.14,95 \%$ CI: $1.01-4.53)$ increased the probabilities of adherence to treatment. Patients who received sequential endocrine treatment showed a higher adherence than those who received one-drug treatment. Women treated with radiotherapy showed a higher probability of adherence $(\mathrm{OR}=1.8,95 \% \mathrm{CI}: 0.8-4.05)$. Women with reported side effects on physician report were two-fold more likely to be adherent than those who did not (OR $=1.81,95 \%$ CI: 0.81-4.02); when adherence was self-reported by patients high, an association was observed with age, with women aged 50-74 years proving significantly more adherent than those aged under 50 years $(\mathrm{OR}=2.75,95 \% \mathrm{CI}: 1.41-5.36)$. The presence of adverse drug effects was not associated with adherence.

Age, endocrine treatment and adjuvant chemotherapy were associated with adherence to treatment measured by the prescription refill of the drug. Women aged 50-74 years and those aged over 74 years showed a higher probability of adherence $(\mathrm{OR}=1.67,95 \% \mathrm{CI}: 1.03-2.70$ and $\mathrm{OR}=1.60,95 \% \mathrm{CI}: 0.76-3.36$, respectively). Similarly, higher probability of adherence with endocrine treatment $(\mathrm{OR}=1.60,95 \% \mathrm{CI}$ : $1.05-2.43)$ was observed for women receiving adjuvant chemotherapy treatment. Patients with aromatase inhibitors only and sequential therapy (tamoxifen + aromatase inhibitors) showed a higher probability of being adherent as compared with those with Tamoxifen only $(\mathrm{OR}=2.0$, 95\% CI: $1.18-3.38$ and OR $=2.27,95 \%$ CI: $1.34-3.84$, respectively). Finally, we observed a non significant increase in probability of adherence in the presence of side effects $(\mathrm{OR}=1.25,95 \% \mathrm{CI}$ : 0.81-1.93).

\section{DISCUSSION}

The results of this study suggest that adherence by BC patients to endocrine treatment over the course of 5 years is a problem that calls for ongoing assessment by physicians in clinical practice. However, while adherence measured by refill prescriptions during the cumulative 5 years of treatment showed $74.7 \%$ adherence, physicians reported much higher adherence $(94.7 \%)$ and patient's $92 . \%$ of adherence. Hence, the variable used to measure adherence is critical when it comes to identifying adherence problems among BC patients.

The range of values observed in our study are consistent with those to be found in the literature (Partridge et al, 2003, 2008; Barron et al, 2007; Ruddy et al, 2009; Verma et al, 2011; Banning, 2012). A recent review of different oral cancer treatments reported persistence of $50-79 \%$ based on refill records (Ruddy et al, 2009). Using similar approaches, other recently published studies indicate lower percentages than those observed by us, that is, cumulative 5-year persistence levels of $49 \%$ in a Dutch cohort (Herk-Sukel et al, 2010) and 68\% in another US population (Hershman et al, 2010a). Self-report measures yielded more inconsistent results: while one study used a single measure to report an adherence figure of 45\% (Atkins and Fallowfield, 2006), another that relied on the self-reported answers of the sample reported adherence of $100 \%$, which then fell to $80 \%$ for tamoxifen and $60 \%$ for anastrozole (Ziller et al, 2009) when drug refills were used as the measure. It is of interest to note that, of all the measures used, it was physicians' reports that indicated the highest adherence levels, which probably implies an overestimation of patients' adherence to treatment (Rudd, 1979; Partridge, 2006). Taking all the observed measures into consideration, our study shows that $5.3-25.3 \%$ of patients failed to follow the prescribed course of oral endocrine treatment. Such non-adherence has clinical consequences in terms of disease prognosis, as was recently shown by a Scottish study which observed a $10 \%$ higher risk of death (hazard ratio $=1.10,95 \%$ CI: 1.001-1.21) associated with adherence to tamoxifen of under $80 \%$, (McCowan et al, 2008) though cumulative 5-year persistence was lower in their case (49\%) than in ours. Similarly, over $-80 \%$ adherence was seen by another paper to be associated with a lower risk of death. Indeed, this $80 \%$ cutoff is clearly supported (Hershman et al, 2010b), to the extent that any decrease in adherence below this point is accompanied by an increase in the risk of death.

However, as mentioned above, one crucial aspect that emerges from the literature is the problem of measuring adherence (Ruddy et al, 2009), and this study allows to consider in a comparative approach. As a background, here is no gold standard for adherence measurement, this fact has as a consequence that there is a diversity of measures used. The fact that our study design made use of different approaches to measure adherence assessed by physician, by the patient and the cumulative 5 years persistence with the refill of the treatment afforded us an opportunity to assess the consistency of the different variables used. Our results clearly 
Table I Description of the clinical variables of patients

\begin{tabular}{|c|c|c|c|c|}
\hline & $\begin{array}{l}\text { Tamoxifen } \\
n(\%)^{\mathbf{a}}\end{array}$ & $\begin{array}{c}\text { Inhibitors } \\
n(\%)^{\mathbf{b}}\end{array}$ & $\begin{array}{c}\text { Tamoxifen }+ \text { inhibitors } \\
n(\%)^{c}\end{array}$ & $\begin{array}{l}\text { Others } \\
n(\%)^{d}\end{array}$ \\
\hline Endocrine therapy & 200 & 206 & 243 & 43 \\
\hline \multicolumn{5}{|l|}{ Age at diagnosis } \\
\hline$\leqslant 49$ years & $110(55.0)$ & $10(4.9)$ & $47(19.3)$ & $16(37.2)$ \\
\hline $50-74$ years & $69(34.5)$ & $143(69.4)$ & $185(76.1)$ & $22(51.2)$ \\
\hline$\geqslant 75$ years & $21(10.5)$ & $53(25.7)$ & II (4.5) & $5(11.6)$ \\
\hline \multicolumn{5}{|l|}{ Stage } \\
\hline 1 & $10 \mid(50.5)$ & $83(40.3)$ & $120(49.4)$ & $17(39.5)$ \\
\hline$\| A$ & $55(27.5)$ & $63(30.6)$ & $69(28.4)$ & $13(30.2)$ \\
\hline$\| B$ & $29(14.5)$ & $29(14.1)$ & $30(12.3)$ & $5(11.6)$ \\
\hline IIIA & $8(4.0)$ & $15(7.3)$ & $15(6.2)$ & $4(9.3)$ \\
\hline TNMx & $7(3.5)$ & $16(7.8)$ & $9(3.7)$ & $4(9.3)$ \\
\hline \multicolumn{5}{|l|}{ Surgical treatment } \\
\hline \multicolumn{5}{|l|}{ Type of surgical treatment } \\
\hline Conservative breast surgery & $149(76.8)$ & | 48 (73.7) & $208(85.6)$ & $31(72.1)$ \\
\hline Radical breast surgery & $45(23.2)$ & $53(26.4)$ & $35(14.4)$ & $12(27.9)$ \\
\hline \multicolumn{5}{|l|}{ Radiotherapy } \\
\hline Yes & $155(77.5)$ & $157(76.2)$ & $216(88.9)$ & $32(74.4)$ \\
\hline No & $45(22.5)$ & $49(23.8)$ & $27(11.1)$ & II (25.6) \\
\hline \multicolumn{5}{|l|}{ Neoadjuvant chemotherapy } \\
\hline Yes & $26(13.0)$ & $10(4.9)$ & $5 \mid(21.0)$ & $7(16.3)$ \\
\hline No & $174(87.0)$ & $196(95.1)$ & $192(79.0)$ & $36(83.7)$ \\
\hline \multicolumn{5}{|l|}{ Adjuvant chemotherapy } \\
\hline Yes & $100(50.0)$ & $73(35.4)$ & I 67 (68.7) & $21(48.8)$ \\
\hline No & $100(50.0)$ & $133(64.6)$ & $76(31.3)$ & $22(51.2)$ \\
\hline \multicolumn{5}{|l|}{ Side effects } \\
\hline Osteopenia & $3(1.5)$ & $21(10.2)$ & $29(11.9)$ & $4(9.3)$ \\
\hline Vaginal dryness & $9(4.5)$ & $7(3.4)$ & $20(8.2)$ & $7(16.3)$ \\
\hline Weight gain & $14(7.0)$ & $4(1.9)$ & $12(4.9)$ & $3(7.0)$ \\
\hline Osteoporosis & $4(2.0)$ & $12(5.8)$ & $13(5.3)$ & $4(9.3)$ \\
\hline Depression & $5(2.5)$ & $10(4.9)$ & $12(4.9)$ & $3(7.0)$ \\
\hline Irregular menstruation & $16(8.0)$ & I (0.5) & $9(3.7)$ & $3(7.0)$ \\
\hline Blood loss & $9(4.5)$ & $4(1.9)$ & $9(3.7)$ & $3(7.0)$ \\
\hline Oedema & $5(2.5)$ & $7(3.4)$ & $11(4.5)$ & $2(4.7)$ \\
\hline Headache & $7(3.5)$ & I (0.5) & $9(3.7)$ & $4(9.3)$ \\
\hline Weakened hair and nails & $2(1.0)$ & $2(1.0)$ & $9(3.7)$ & I (2.3) \\
\hline Nausea/vomiting & $6(3.0)$ & $5(2.4)$ & $2(0.8)$ & I (2.3) \\
\hline Diarrhoea & $2(1.0)$ & I (0.5) & $8(3.3)$ & $3(7.0)$ \\
\hline Insomnia & $4(2.0)$ & 0 & $8(3.3)$ & I (2.3) \\
\hline Hypercholesterolaemia & 0 & $3(1.5)$ & $6(2.5)$ & 0 \\
\hline Memory loss & $2(1.0)$ & $2(1.0)$ & $3(1.2)$ & $2(4.7)$ \\
\hline Vision changes & $2(1.0)$ & 0 & $2(0.8)$ & I (2.3) \\
\hline Deep vein thrombosis & I $(0.5)$ & I (0.5) & $2(0.8)$ & I (2.3) \\
\hline Difficulty concentrating & 0 & 0 & $2(0.8)$ & I (2.3) \\
\hline Bone fracture & 0 & I (0.5) & I $(0.4)$ & I (2.3) \\
\hline Stroke & 0 & $2(1.0)$ & 0 & I (2.3) \\
\hline Pulmonary embolism & 0 & I $(0.5)$ & I (0.4) & I (2.3) \\
\hline Cardiotoxicity & 0 & 0 & I (0.4) & 0 \\
\hline
\end{tabular}

${ }^{a}$ Difference from the total $N=200$, corresponding to missing values. ${ }^{b}$ Difference from the total $N=206$, corresponding to missing values. ${ }^{c}$ Difference from the total $N=243$, corresponding to missing values. ${ }^{d}$ Difference from the total $N=43$, corresponding to missing values. 
Table 2 Description of adherence measures

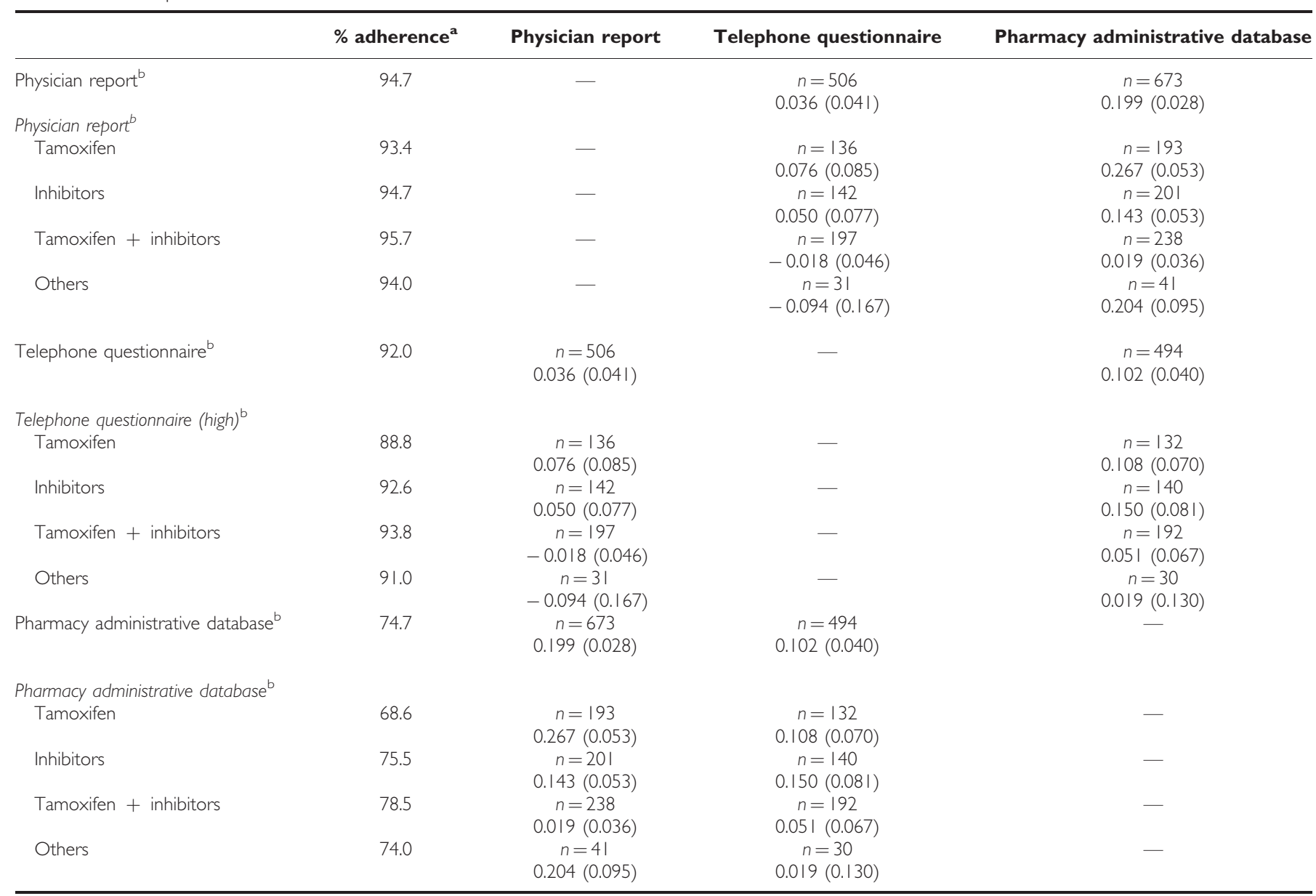

a\% Adherence adjusted for centre, age at diagnosis and stage. ${ }^{\mathrm{b} C o n c o r d a n c e: ~ k a p p a ~(s . e . ~ f o r ~ k a p p a ~ s t a t i s t i c) . ~}$

showed a very low degree of concordance among the different measures, (Osterberg and Blaschke, 2005; Ruddy et al, 2009). Selfreported adherence is known to overestimate the true values, when compared with other more reliable measures, such as electronic control of medical administration (Waterhouse et al, 1993; Escalada and Griffiths, 2006; Shi et al, 2010). Social desirability bias and recall bias may account for this (Ruddy et al, 2009). A remarkable result in our study was the low concordance observed between physician and self-reported adherence, suggesting a lack of consistent criteria among physicians and patients in evaluating adherence, even though physicians clearly rated adherence higher than did any other alternative measure. In all likelihood, physicians only report a patient as being non-adherent when the problem is evident and openly acknowledged during the consultation, conceivably linked to assessment of adverse effects in some cases. Although a low probability of false positives is thus to be expected in such reports, physicians might nonetheless detect a small percentage of patients with adherence problems.

The explanation for this lack of concordance among the measures used may lie, to a significant degree, in the different objectives and sources of information for each measure. Whereas physicians assess adherence by an evaluative perception of problems clearly and openly manifested by the patient, such as lack of response, side effects or questions posed by either one; pharmacy records, on the other hand, assess cumulative 5 years persistence as measure of adherence on the basis of an administrative database that collects information of which patients are not aware, via a process free of intervention by health professionals; thereby making it the most reliable and objective indicator. An accepted assumption in the administrative records is that drug refills are associated with adherence (assuming pill intake), especially in the case of long lasting treatments such as oral endocrine therapy for BC (Cramer et al, 2008). Furthermore, the use of telephone interviews to measure self-reported adherence probably led to potential social desirability bias, with an ensuing overestimation of adherence. Even so, findings from this study do not allow for a reference method to be selected because results would need to be checked against biological data that are currently unavailable. However, the prescription refill is probably the most reliable method in this context of long-term therapy.

The relationship between explanatory variables and adherence displayed relevant variation, depending on the adherence measure considered. Based on physician report, increasing age was associated with a higher level of treatment adherence, after adjusting for all relevant variables, which is in line with previous studies in the literature (Partridge et al, 2003; Barron et al, 2007). An interesting finding is the fact that adjuvant chemotherapy was associated with a higher probability of adherence. This is consistent with a previous cohort study, which also used persistence as an outcome variable in conjunction with a 4.5 -year follow-up period (Hershman et al, 2010a). A possible explanation for this finding might be the intensity of therapy as perceived by the patient and the higher interaction with the physician, which such treatment necessarily implies. The lack of association 
Table 3 Prevalence and odds ratio (OR) associated with adherence for each of the methods used

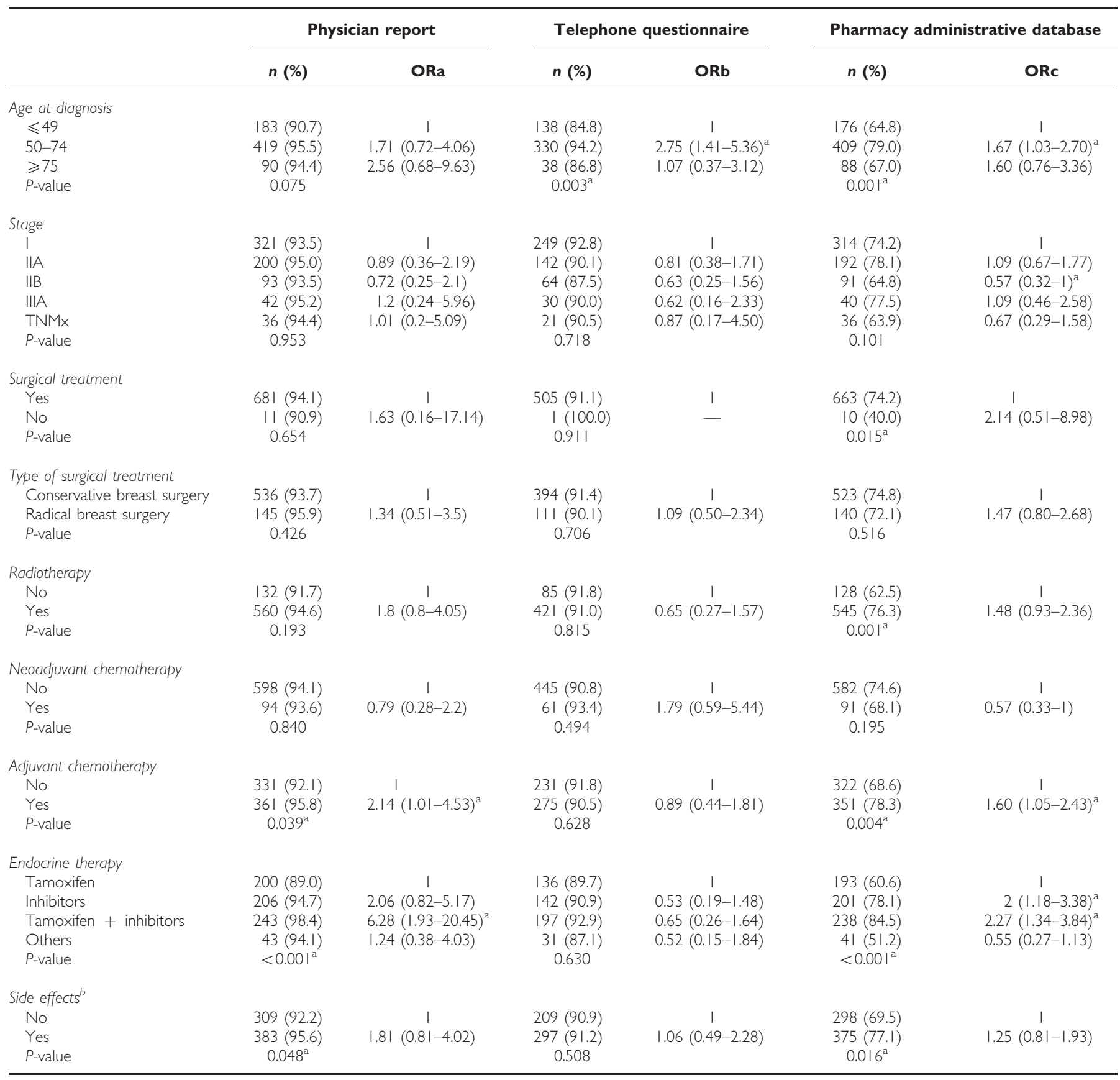

Abbreviations: ORa = adjusted for centre, age at diagnosis, adjuvant chemotherapy, adverse effects and endocrine therapy; ORb = adjusted for centre, age at diagnosis; $\mathrm{ORc}=$ adjusted for centre, age at diagnosis, adjuvant chemotherapy, radiotherapy, side effects and endocrine therapy. $n(\%)=$ number of cases (\% adherence); $P$-value: $\chi^{2}$-test. ${ }^{a}$ Statistical significance, $P<0.05$. ${ }^{b}$ Adverse effects of physician report.

between adverse effects and non-adherence is unexpected; several papers have reported this finding (Grunfeld et al, 2005; Atkins and Fallowfield, 2006; Hershman et al, 2010a), which we failed to observe; although it has been found non differential adherence in clinical trials between treatment and placebo group (Fisher et al, 1996); indicating that the relationship is more complex than usually assumed. However, it should be stressed again that these findings are based on physician report, which could bias the relationship. Finally, when compared with one-drug treatment, sequential oral endocrine therapy was associated with an increased probability of adherence. This could be relevant, bearing in mind the Catalonian guideline recommends the adoption of this therapeutic approach (Manchon et al, 2010) and a progressive reduction in the indication of one-drug treatment in endocrine therapy for early BC.

It is interesting to notice that the results observed are quite consistent with the physician report when the multivariate analysis is analysed using refill prescriptions as dependent variable. Older age, adjuvant chemotherapy, sequential treatment or aromatase inhibitors alone as endocrine treatment in comparison to tamoxifen alone were significantly associated with higher probability of adherence. These results increased the consistency of the observations regarding the relevant variables associated with adherence. Here, it is worth mentioning the fact the adjuvant 
radiotherapy was also associated with adherence, which adds support to the fact that intense treatment and its correlate of visits with professionals and health care contacts could be a possible explanation for this finding. Interestingly, side effects showed no significant relationship with adherence although a higher percentage of adverse effects was associated with higher adherence in bivariate relationship. The explanation may possibly lie in the association described by patients between the drug's perceived efficacy and the presence of adverse effects (Bell, 2009). Focusing on patients' self-reports, only age was significantly associated with adherence. The role of age is consistent with other measures used here and in other studies (Ruddy et al, 2009), as well as the lack of a clear pattern with other demographic and socio-economic variables. This lack of association with variables other than age prevents a profile of patients with a higher risk of non-adherence from being drawn up.

Some limitations of this study should be considered. It was a retrospective study with a 5-year follow-up of refill rates, and subjects' answers to questionnaire might have been influenced by recall bias. Furthermore, as physicians' reports were based on clinical records, there is the potential problem of possible omissions of patients' non-adherence. Although the use of an administrative database aimed at reimbursing the cost of drugs to the public health care system raises the point that the database was never intended for such a purpose, this should nevertheless be counterbalanced by the fact that patients' lack of awareness and the database's mandatory nature probably serve to make its content highly reliable in terms of prescription-refill rates. Moreover, this database allowed for a 5-year follow-up, which was not the case in several previous studies (Lebovits et al, 1990; Waterhouse et al, 1993; Murthy et al, 2002; Partridge et al, 2003; Atkins and Fallowfield, 2006; Barron et al, 2007; Thompson et al, 2007). In fact, relevant aspect of our study was its inclusion of all

\section{REFERENCES}

Andersson K, Melander A, Svensson C, Lind O, Nilsson JL (2005) Repeat prescriptions: refill adherence in relation to patient and prescriber characteristics, reimbursement level and type of medication. Eur J Public Health 15: 621-626

Atkins L, Fallowfield L (2006) Intentional and non-intentional nonadherence to medication amongst breast cancer patients. Eur J Cancer 42: 2271-2276

Banning M (2012) Adherence to adjuvant therapy in post-menopausal breast cancer patients: a review. Eur J Cancer Care 21: 10-19

Barron TI, Connolly R, Bennett K, Feely J, Kennedy MJ (2007) Early discontinuation of tamoxifen: a lesson for oncologists. Cancer 109: $832-839$

Bell K (2009) 'If it almost kills you that means it's working!' Cultural models of chemotherapy expressed in a cancer support group. Soc Sci Med 68: 169-176

Berry DA, Cronin KA, Plevritis SK, Fryback DG, Clarke L, Zelen M, Mandelblatt JS, Yakovlev AY, Habbema JD, Feuer EJ (2005) Effect of screening and adjuvant therapy on mortality from breast cancer. $\mathrm{N} \mathrm{Engl} \mathrm{J}$ Med 353: 1784-1792

Borras JM, Espinas JA, Ferro T, Ribes J, Clèries R, Aliste L, Corral J, Escribà JM, Esteban L, Font R, Gálvez J, Manchon P, Melià A, Pareja L, Prades JLl, Roca L, Sanz X (2009) Cancer strategy in Catalonia, Spain. Clin Transl Oncol 11: 1-30

Borras JM, Sanchez-Hernandez A, Navarro M, Martinez M, Mendez E, Ponton JL, Espinas JA, Germa JR (2001) Compliance, satisfaction, and quality of life of patients with colorectal cancer receiving home chemotherapy or outpatient treatment: a randomised controlled trial. BMJ 322: 826

Cramer JA, Roy A, Burrell A, Fairchild CJ, Fuldeore MJ, Ollendorf DA, Wong PK (2008) Medication compliance and persistence: terminology and definitions. Value Health 11: 44-47

Darkow T, Henk HJ, Thomas SK, Feng W, Baladi JF, Goldberg GA, Hatfield A, Cortes J (2007) Treatment interruptions and non-adherence with imatinib and associated healthcare costs: a retrospective analysis among treatment regimens for oral endocrine therapy. Finally, because of sample size, it has not been possible to explore in depth the role of individual adverse effects on adherence.

In conclusion, non-adherence was found to be a relevant health problem among BC patients on oral endocrine therapy. About three quarters of all study subjects fully completed the 5-year treatment recommended in the clinical guidelines. Although young age at diagnosis was the variable most clearly associated with nonadherence to treatment, sequential as opposed to one-drug therapy, adjuvant chemotherapy and radiotherapy were all associated with a higher probability of adherence with treatment. A relevant result of this study, however, was the lack of agreement among the different measures of adherence, the reason for which probably goes beyond the fact that they might be measuring different elements of a similar concept or be drawn from different data sources. The relevant question remains the need to assume that the adherence in daily clinical practice depends on how we measure it.

\section{ACKNOWLEDGEMENTS}

We should like to thank the following: all data managers who were involved in the data-collection process; Yolanda Benavente and Luisa Aliste for their help with the statistical analysis; and Meritxell Nomen for her administrative support throughout the study. This project was partially supported by grants from the Carlos III Institute of Health (FIS 09FIS039; PI11/02011) and the Spanish Cancer Research Network (RTICC 06/0089)

\section{Conflict of interest}

The authors declare no conflict of interest. managed care patients with chronic myelogenous leukaemia. Pharmacoeconomics 25: 481-496

DiMatteo R (2004) Variations in patient adherence to medical recommendations: a quantitative review of 50 years of research. Med Care 42: 200-209

Early Breast Cancer Trialist' Collaborative Group (EBCTCG) (2005) Effects of chemotherapy and hormonal therapy for early breast cancer on recurrence and 15-year survival: an overview of the randomised trials. Lancet 365: 1687-1717

Escalada P, Griffiths P (2006) Do people with cancer comply with oral chemotherapy treatments? Br J Community Nurs 11: 532-536

Fallowfield L, Atkins L, Catt S, Cox A, Coxon C, Langridge C, Morris R, Price M (2006) Patients' preference for administration of endocrine treatments by injection or tablets: results from a study of women with breast cancer. Ann Oncol 17: 205-210

Fisher B, Dignam J, Bryant J, Wolmark N (1996) Five versus more than five years of tamoxifen therapy for breast cancer patients with negative lymph nodes and estrogen receptor positive tumors. JNCI 88: 1529-1542 Grunfeld EA, Hunter MS, Sikka P, Mittal S (2005) Adherence beliefs among breast cancer patients taking tamoxifen. Patient Educ Couns 59: 97-102

Haynes RB, Sackett DL, Taylor DW, Roberts RS, Johnson AL (1977) Manipulation of the therapeutic regimen to improve compliance: conceptions and misconceptions. Clin Pharmacol Ther 22: 125-130

Herk-Sukel MP, van de Poll-Franse LV, Voogd AC, Nieuwenhuijzen GA, Coebergh JW, Herings RM (2010) Half of breast cancer patients discontinue tamoxifen and any endocrine treatment before the end of the recommended treatment period of 5 years: a population-based analysis. Breast Cancer Res Treat 122: 843-851

Hershman DL, Kushi LH, Shao T, Buono D, Kershenbaum A, Tsai WY, Fehrenbacher L, Lin GS, Miles S, Neugut AI (2010a) Early discontinuation and nonadherence to adjuvant hormonal therapy in a cohort of 8,769 early-stage breast cancer patients. J Clin Oncol 28: 4120-4128

Hershman DL, Shao T, Kushi LH, Buono D, Tsai WY, Fehrenbacher L, Kwan M, Gomez SL, Neugut AI (2010b) Early discontinuation and 
non-adherence to adjuvant hormonal therapy are associated with increased mortality in women with breast cancer. Breast Cancer Res Treat 126(2): 529-537

Landis JR, Koch GG (1977) The measurement of observer agreement for categorical data. Biometrics 33: 159-174

Lash TL, Fox MP, Westrup JL, Fink AK, Silliman RA (2006) Adherence to tamoxifen over the five-year course. Breast Cancer Res Treat 99: 215-220

Lebovits AH, Strain JJ, Schleifer SJ, Tanaka JS, Bhardwaj S, Messe MR (1990) Patient noncompliance with self-administered chemotherapy. Cancer 65: 17-22

Liu G, Franssen E, Fitch MI, Warner E (1997) Patient preferences for oral versus intravenous palliative chemotherapy. J Clin Oncol 15: 110-115

Manchon P, Borras JM, Ferro T, Espinas JA, Breast Cancer OncoGuia Group (2010) Breast Cancer OncoGuia. Clin Transl Oncol 12: 113-138

McCowan C, Shearer J, Donnan PT, Dewar JA, Crilly M, Thompson AM, Fahey TP (2008) Cohort study examining tamoxifen adherence and its relationship to mortality in women with breast cancer. Br J Cancer 99: $1763-1768$

Mouridsen H, Giobbie-Hurder A, Goldhirsch A, Thurlimann B, Paridaens R, Smith I, Mauriac L, Forbes JF, Price KN, Regan MM, Gelber RD, Coates AS (2009) Letrozole therapy alone or in sequence with tamoxifen in women with breast cancer. $N$ Engl J Med 361: 766-776

Murthy V, Bharia G, Sarin R (2002) Tamoxifen non-compliance: does it matter? Lancet Oncol 3: 654

Nilsson JL, Andersson K, Bergkvist A, Bjorkman I, Brismar A, Moen J (2006) Refill adherence to repeat prescriptions of cancer drugs to ambulatory patients. Eur J Cancer Care 15: 235-237

Osterberg L, Blaschke T (2005) Adherence to medication. N Engl J Med 353: 487-497

Partridge AH (2006) Non-adherence to endocrine therapy for breast cancer. Ann Oncol 17: 183-184
Partridge AH, LaFountain A, Mayer E, Taylor BS, Winer E, Asnis-Alibozek A (2008) Adherence to initial adjuvant anastrozole therapy among women with early-stage breast cancer. J Clin Oncol 26: 556-562

Partridge AH, Wang PS, Winer EP, Avorn J (2003) Nonadherence to adjuvant tamoxifen therapy in women with primary breast cancer. J Clin Oncol 21: 602-606

Rudd P (1979) In search of the gold standard for compliance measurement. Arch Intern Med 139: 627-628

Ruddy K, Mayer E, Partridge A (2009) Patient adherence and persistence with oral anticancer treatment. CA Cancer J Clin 59: 56-66

Shi L, Liu J, Koleva Y, Fonseca V, Kalsekar A, Pawaskar M (2010) Concordance of adherence measurement using self-reported adherence questionnaires and medication monitoring devices. Pharmacoeconomics 28: $1097-1107$

Thompson AM, Dewar J, Fahey T, McCowan C (2007) Association of poor adherence to prescribed tamoxifen with risk of death from breast cancer. Society of Clinical Oncology Breast Cancer Symposium; San Francisco, California (abstract No. 130)

Verma S, Madarnas Y, Shedev S, Martin G, Bajcar J (2011) Patient adherence to aromatase inhibitor treatment in the adjuvant setting. Curr Oncol 11: S3-S9

Wang PS, Benner JS, Glynn RJ, Winkelmayer WC, Mogun H, Avorn J (2004) How well do patients report noncompliance with antihypertensive medications?: a comparison of self-report versus filled prescriptions. Pharmacoepidemiol Drug Saf 13: 11-19

Waterhouse DM, Calzone KA, Mele C, Brenner DE (1993) Adherence to oral tamoxifen: a comparison of patient self-report, pill counts, and microelectronic monitoring. J Clin Oncol 11: 1189-1197

Ziller V, Kalder M, Albert US, Holzhauer W, Ziller M, Wagner U, Hadji P (2009) Adherence to adjuvant endocrine therapy in postmenopausal women with breast cancer. Ann Oncol 20: 431-436

This work is published under the standard license to publish agreement. After 12 months the work will become freely available and the license terms will switch to a Creative Commons Attribution-NonCommercial-Share Alike 3.0 Unported License. 